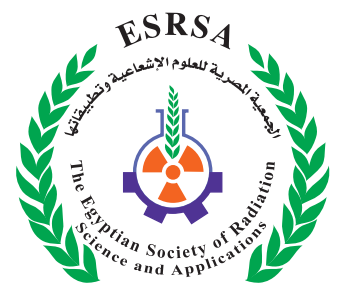

\title{
Examination of Quality Control Parameters in some Diagnostic X-ray Units
}

\author{
Alfawwal, T.M. ${ }^{1,2 *}$ Kutbi, R.A ${ }^{3}$
}

Received: 14/05/2019

Accepted: 03/07/2019

E-mail: tahaalfawwal@hotmail.com

\section{KEYWORDS}

Quality Control, Reproducibility, Accuracy, Linearity and Diagnostic X-ray, RaySafe.

\section{ABSTRACT}

Periodical performance of quality control in diagnostic x-ray machines is important to provide minimum dose delivered to patients and high image quality. The current study is aimed to assure the quality control of conventional X-ray machines over some Makkah city hospitals according to quality control protocol and the measured parameters values are compared to the international acceptance limits. Some factors affecting on quality assurance of conventional X-ray machines such as reproducibility of time, applied high voltage, kilo-voltage accuracy, time accuracy, linearity and half value layer of X-ray machines over four hospitals in Makkah city are investigated. These parameters are measured by using the RaySafe solid-state detector connected with suitable ionization chambers located at $100 \mathrm{~cm}$ source to image detector. Time Reproducibility ranged from $0.05 \%$ to $0.07 \%$ and high voltage reproducibility ranged from $0.09 \%$ to $0.14 \%$. Kilo-voltage accuracy ranged from 0.22 to $3.5 \%$ and time accuracy ranged from 0.02 to $4.1 \%$ respectively. Coefficients of linearity of diagnostic X-ray units in four hospitals in KSA were $0.02,0.004,0.02$ and 0.24 . This study concluded that quality control examinations are within the tolerance limit of the American Association of Physicist in Medicine, "(AAPM)". It assists in the optimization of a dose delivered to patients.

1. Physics Department, Faculty of Applied Sciences, Umm Al-Qura University, KSA, P. Box 21955.

2. Radiation Protection Department, Nuclear Research Center, Atomic Energy Authority, Cairo. P.O.13759, Egypt.

3. The radiology department in KAMC-Jeddah, Department of Medical Physics. 


\section{INTRODUCTION}

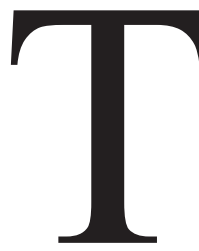

he main objectives of quality assurance of an X-ray machine are to obtain high accuracy of the operating diagnostic X-ray and to optimize of the radiation dose delivered to patient. Quality control tests that are routinely carried out include: reproducibility of tube voltage, dose output, time, $\mathrm{kVp}$ accuracy, time accuracy, half value layer, the relationship between incidence air kerma and $X$-ray tube current, in addition to the linearity test (a relationship between $\mathrm{X}$-ray tube output and $\mathrm{kVp}$ ). The quality control test methods used in the present research are in agreement with the American Association of Physics in Medicine (AAPM, 1981) published quality control protocol for radiological equipment. The quality of X-rays in terms of the half value layer is considered as a part of quality assurance (Stephen et al., 2001 and Begum et al., 2011). The dose output reproducibility is studied based on the procedures for measuring entrance skin exposure (ESE) following American Association of Physics in Medicine (AAPM, 1991) report. Quality control tests for X-ray are studied based on the American Association of Physics in Medicine report (AAPM, 1981 and Plotti, 1995).

Our aim in this study is to investigate some factors affecting the quality assurance of four radiographic $X$-ray units in Makkah city. These factors include reproducibility for dose output, time and applied high voltage, the accuracy of kilo-voltage, tube current, time accuracy, the relationship between $X$ ray tube output and $\mathrm{kV}$.

\section{MATERIALS AND METHODS}

The RaySafe Xi, Fluke Corporation, The USA (America) manufacturer is used to perform the quality control tests for four radiographic $\mathrm{x}$-ray equipments. Reproducibility for dose output, time and applied high voltage, the accuracy of kilo-voltage, tube current, time accuracy, linearity, the relationship between $\mathrm{X}$-ray tube output and $\mathrm{kV}$ are explained as follows:

\section{The Reproducibility}

Reproducibility for dose output applied $\mathrm{kV}$ and exposure time of radiographic X-ray machine in four hospitals, King Abdullah Medical City Hospital KA$\mathrm{MCH}$, Hera General Hospital (HGH), King Faisal Hospital (KFH) and Maternity \& Children Hospital $(\mathrm{MCH})$ is measured using Unfors RaySafe meter connected to a solid state detector. Measurements are carried on the position of a phantom for the desired field size and for three different exposures are made.

The coefficient of variation is calculated using the next equation 1 (FDA,1999)

\section{$\operatorname{COV}=\frac{\sigma}{\text { avg }} X 100$}

Where: Calculated value of standard deviation $[\sigma]$, of dose $[\mathrm{mGy}]$, time $[\mathrm{ms}]$ or voltage $[\mathrm{kV}]$. Calculated value of mean, avg of dose [mGy], time[ms] or voltage $[\mathrm{kV}]$.

\section{Accuracy}

The accuracy or relative percentage error of tube voltage and time setting is examined for each machine. Three exposures are recorded for tube voltage and irradiated time. The relative percentage error is calculated using the equation 2 .

Relative $\%$ error $=\frac{\text { Measured } k V-\text { Accepted } k V}{\text { Accepted } k V} \times 100$ (2)

Where: Accepted or assigned a value of the value of time $[\mathrm{ms}]$ or voltage $[\mathrm{kV}]$, that is entered digitally into a control panel. The measured value of time $[\mathrm{ms}]$ or voltage $[\mathrm{kV}]$ that is recorded using Ray Safe meter. The acceptance tolerance limit of the American Association of Physicist in Medicine recommendation (AAPM, 2002) and National New Zealand Laboratory, (Plotti, 1995). 


\section{The dose output linearity}

The dose output linearity is calculated by using the equation (3) (FDA, 1999).

$\frac{\mathrm{X} 2-\mathrm{X} 1}{\mathrm{X} 2+\mathrm{X} 1}<0.1$

Where $X_{1}$ and $X_{2}$ are two successive readings of dose output in the unit of $\mu \mathrm{Gy} / \mathrm{mAs}$. A relation chip between applied kilo-voltage and the dose output is studied for four radiographic x-ray units in four hospitals in Makkah city. The physical X-ray machine is adjusted at $10 \mathrm{mAs}$ and $500 \mathrm{msec}$. The source to image distance (SID) is adjusted at $100 \mathrm{~cm}$ where the ionization chamber of the Unfors Ray Safe is located in center beam axis to avoid heel effect. The dose output factors for each x-ray machine is generated and used for dose estimation (Taha et al., 2014).

\section{RESULTS}

\section{Reproducibility}

Reproducibility for dose output applied $\mathrm{kV}$ and exposure time for x-ray machine in King Abdullah Medical City Hospital KAMCH, GE X-ray machines in three hospitals, Hera General Hospital (HGH), King Faisal Hospital (KFH), Maternity \& Children Hospital (MCH) were presented as shown in (table 1).

Table (1) : Reproducibility of $k V$, time and dose output for four X-ray machines in four medical centers, $\mathrm{KAMCH}, \mathrm{MCH}, \mathrm{HGH}$, and $\mathrm{KFH}$ Reproducibility of $\mathrm{kV}$, time and dose output for four X-ray ma chines in four medical centers, $K A M C H, M C H, H G H$, and $K F H$.

\begin{tabular}{|c|c|c|c|c|}
\hline \multirow{2}{*}{ Hospital. } & FFD & \multicolumn{3}{|c|}{ Reproducibility (CV\%) } \\
\cline { 2 - 5 } & & $\mathbf{k V p}$ & Time (s) & Dose (mGy) \\
\hline KAMC & $100 \mathrm{~cm}$ & $\mathbf{0 . 0 9}$ & 0.07 & 0.34 \\
\hline MCH & $100 \mathrm{~cm}$ & 0.07 & 0.05 & 0.14 \\
\hline HGH & $100 \mathrm{~cm}$ & 0.09 & 0.07 & 0.44 \\
\hline KFH & $100 \mathrm{~cm}$ & 0.14 & 0.06 & 1.29 \\
\hline
\end{tabular}

Reproducibility for kilovoltage for $\mathrm{x}$-ray machines was varied from $0.07 \%$ to $0.14 \%$, of time was varied from $0.05 \%$ to $0.07 \%$ and dose output was varied from $0.14 \%$ to $1.29 \%$ that is lower than the tolerance level (5\%) (FDA, 1999).

\section{$k V$ and Time accuracy}

kVp Accuracy for King Abdullah Medical City Hospital (KAMCH), Hera General Hospital (HGH), King Faisal Hospital (KFH), Maternity \& Children Hospital $(\mathrm{MCH})$ of different settings of four X-ray machines was determined. It examined for kilovoltage setting from $50-100 \mathrm{kV}, 20 \mathrm{mAs}$. Time accuracy for King Abdullah Medical City Hospital (KA$\mathrm{MCH})$, Hera General Hospital (HGH), King Faisal Hospital (KFH), Maternity \& Children Hospital
$(\mathrm{MCH})$ of four X-ray machines determined as presented in (Table 2).

Kilo-voltage accuracy and time accuracy for King Abdullah Medical City Hospital (KAMCH), Hera General Hospital (HGH), King Faisal Hospital (KFH), Maternity \& Children Hospital (MCH) were within the kilovoltage and time accuracy of $5 \%$ that issued by (AAPM, 1991; Plotti, 1995). The obtained results were close to the data set published by (Taha, 2015 and Ismail, 2015) and was lower than tolerance $( \pm 10 \%)$ (Plotti,1995).

\section{mAs Linearity}

Coefficient of Linearity of X-ray machine in King Abdullah Medical City Hospital (KAMCH), Hera General Hospital (HGH), King Faisal Hospital 
(KFH), Maternity \& Children Hospital (MCH) was examined using $80 \mathrm{kVp}$ and mAs vary from 2-32
$\mathrm{mAs}$ at $100 \mathrm{~cm}$ FFD and linearity coefficient is calculated for each machine as presented in (table 3 ).

Table (2) : kVp Accuracy at source to image detector $100 \mathrm{~cm}$ and $12 \mathrm{mAs}$.

\begin{tabular}{|c|c|c|c|}
\hline Hospital & Machine Manufacture & $\begin{array}{c}\text { Mean KVp \% } \\
\text { Accuracy range }\end{array}$ & $\begin{array}{c}\text { Mean time \% Accuracy } \\
\text { range }\end{array}$ \\
\hline KAMC & Siemens & $1.50-3.5$ & $0.46-4.1$ \\
\hline MCH & GE & $0.22-1.02$ & $1.44-2$ \\
\hline HGH & GE & $0.12-0.2$ & $002-0.5$ \\
\hline KFH & GE & $0.6-194$ & $0.02-0.11$ \\
\hline
\end{tabular}

Table (3) : Coefficient of Linearity of X-ray machine in King Abdullah Medical City Hospital (KAMCH), Hera General Hospital (HGH), King Faisal Hospital (KFH), Maternity \& Children Hospital $(\mathrm{MCH})$ at $\mathrm{kV}=81, \mathrm{SID}=100$, time $=100 \mathrm{msec}$.

\begin{tabular}{|c|c|}
\hline Hospital/Machine No. & Linearity Coefficient \\
\hline KAMCH & 0.020 \\
\hline MCH & 0.004 \\
\hline HGH & 0.020 \\
\hline KFH & 0.024 \\
\hline
\end{tabular}

Coefficient of linearity of X-ray machine in King Abdullah Medical City Hospital (KAMCH), Hera General Hospital (HGH), King Faisal Hospital (KFH), Maternity \& Children Hospital $(\mathrm{MCH})$ was $0.020,0.004,0.020$ and 0.024 which lower than 0.1 that reported by (Plotti, 1995). It means that the mAs was directly proportional with the dose output at any setting points.

\section{Half Value Layer (HVL).}

The half value layer (HVL) of the x-ray beam was calculated by plotting relative attenuation percentage with and without lead sheet layer versus thickness $(\mathrm{mm})$ for the lead. it was measured at 70 $\mathrm{kV}$ and $32 \mathrm{mAs}$ and source to detector distance 66 $\mathrm{cm}$. Half value layers were calculated as shown in (table 4).

Table (4) : Minimum HVL requirement for an X-ray unit.

\begin{tabular}{|c|c|}
\hline Machine No. & Half Value Layer, $\mathrm{mm}$ \\
\hline KAMCH & 2.9 \\
\hline HCM & 2.9 \\
\hline HGH & 2.9 \\
\hline KFH & 3.0 \\
\hline
\end{tabular}

All half value layers are exceeding the minimum value, $2.3 \mathrm{~mm} \mathrm{Al}$ at $70 \mathrm{kV}$ as an accepted value of (FDA, 1999). The tube voltage and tube output for a diagnostic X-ray machine.
The relationship between X-ray Air kerma $\mu \mathrm{Gy} /$ $\mathrm{mAs}$ and applied tube voltages from 40 to $130 \mathrm{kV}$ at $10 \mathrm{mAs}$ and 500 million seconds of the X-ray machines in the $\mathrm{KAMC}, \mathrm{HGH}, \mathrm{MCH}$, and $\mathrm{KFH}$ was plotted in figures (1-4). 


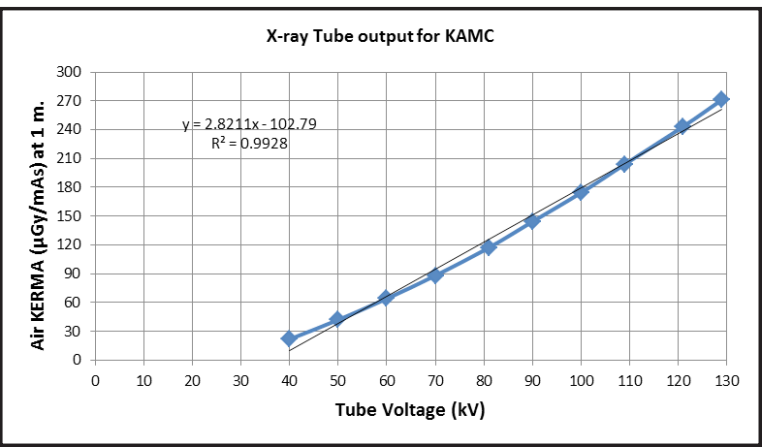

Fig. (1): Tube voltage and tube output for a diagnostic X-ray machine in KAMC.

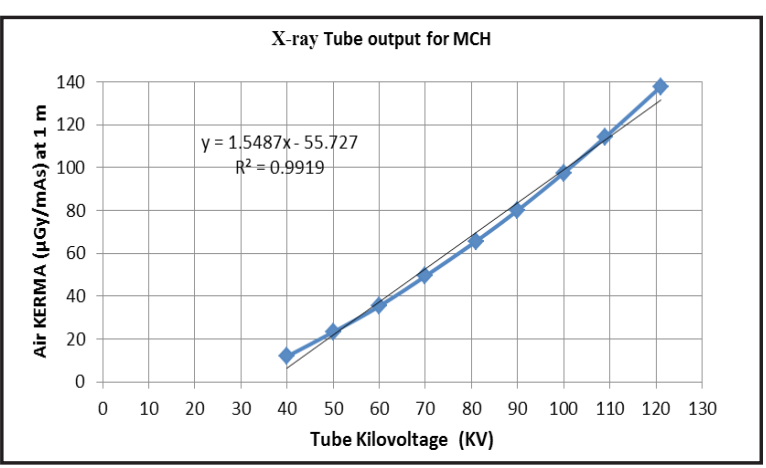

Fig. (3): Tube Voltage (kV) against Air KERMA in the $\mathrm{MCH}$.

The fitted equation for the relationship between tube voltage $(\mathrm{kV})$ against air kerma in the KAMC, the $\mathrm{KAMC}, \mathrm{HGH}, \mathrm{MCH}$, and $\mathrm{KFH}$ was presented in equations (1-4) respectively.so one body can estimate the dose out, $\mu \mathrm{Gy} / \mathrm{mAs}$ for each tube voltage for x-ray tubes in the four radiology departments in the interested hospital as presented in the empirical equations (4-7).

$$
\begin{aligned}
& Y=2.8211 X-102.79 \\
& Y=1.245 X-46.727 \\
& Y=1.5487 X-55.727 \\
& Y=1.2903 X-32.991
\end{aligned}
$$

\section{Where :}

$Y: \quad$ is the air kerma $(\mu G y / m A s)$ at one meter

$X: \quad$ Tube voltage in $\mathrm{kV}$

\section{DISCUSSION}

Reproducibility for kilovoltage for X-ray machines was varied from $0.07 \%$ to $0.14 \%$, of time was

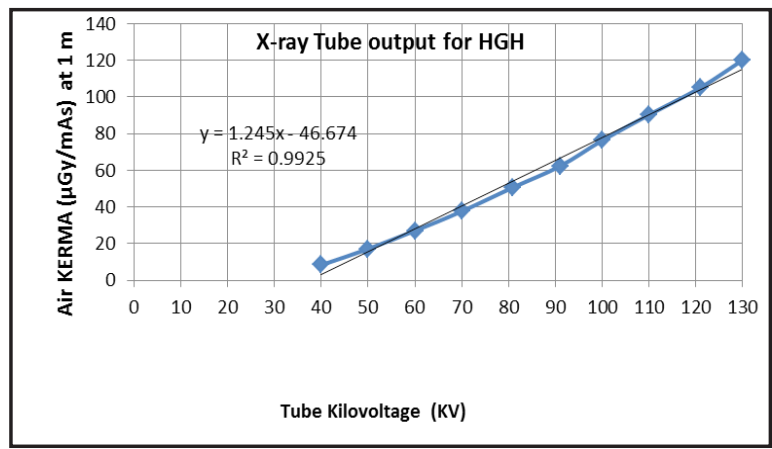

Fig. (2): Tube Voltage (kV) against Air KERMA in the $\mathrm{HGH}$.

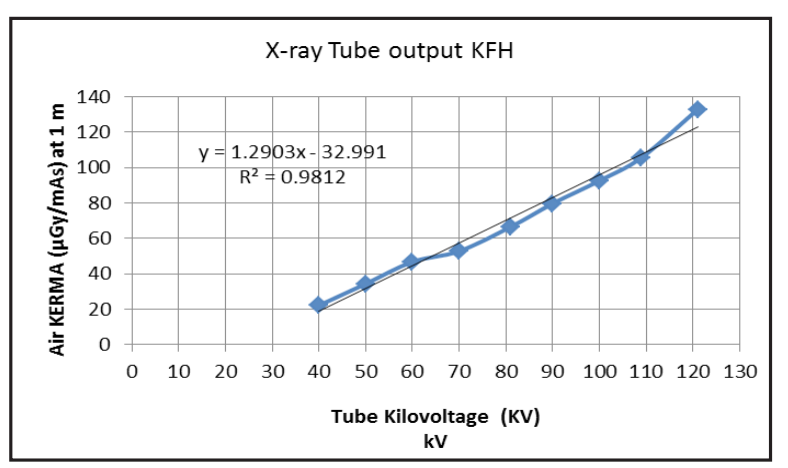

Fig. (3): Tube Voltage (kV) against Air KERMA in the $\mathrm{MCH}$.

varied from $0.05 \%$ to $0.07 \%$ and dose output was varied from $0.14 \%$ to $1.29 \%$ that is lower than the tolerance level ( 5\%) (FDA, 1999), American Association of Physics in Medicine (AAPM, 1981 and Plotti,1995).

Kilo-voltage accuracy and time accuracy for King Abdullah Medical City Hospital (KAMCH), Hera General Hospital (HGH), King Faisal Hospital (KFH), Maternity \& Children Hospital (MCH) were within the kilovoltage and time accuracy of $5 \%$ that issued by (Plotti,1995). The obtained results were close to the data set published by (Taha, 2015 and Ismail, 2015) and was lower than tolerance $( \pm 10 \%)$ (Plotti, 1995).

Coefficient of linearity of X-ray machine in King Abdullah Medical City Hospital (KAMCH), Hera General Hospital (HGH), King Faisal Hospital (KFH), Maternity \& Children Hospital (MCH) was $0.020,0.004,0.020$ and 0.024 which lower than 0.1 
that reported by (Plotti, 1995). It means that the mAs was directly proportional with the dose output at any setting points. All half value layers are exceeding the minimum value, $2.3 \mathrm{~mm} \mathrm{Al}$ at $70 \mathrm{kV}$ as an accepted value of FDA, (FDA, 1999). Half value layers were constant at energies close to that value given by FDA (1999). So HVL has an ability to prevent the hazard of soft X-ray by reducing the entrance skin doses during X-ray imaging. HVL is expected of $\mathrm{X}$-ray tube (age), voltage waveform (Begum et al., 2011). The dose out, $\mu \mathrm{Gy} / \mathrm{mAs}$ for each tube voltage for X-ray tubes in the four radiology departments in the interested hospital can be calculated via the empirical equations and used in dose assessment.

\section{CONCLUSION}

The quality assurance for radiographic X-ray plays an important role in obtain accurate and timely diagnosis. As the kilo-voltage increases by one the air kerma output increases by 2.83, 1.245, 1.548 and 1.290 at $\mathrm{KAMC}, \mathrm{HGH}, \mathrm{MCH}$ and $\mathrm{KFH}$ respectively as presented in figures (1-4). The dose output for each $\mathrm{x}$-ray tube is used as input factor in mathematical patient dose calculation.

\section{ACKNOWLEDGEMENT}

This work is supported by grant No. 55-35 from king Abdelaziz City for science and technology (KACST), Riyad. KSA.

\section{REFERENCES}

- American Association of Physicists Medicine, AAPM. (1981): Basic Quality Diagnostic Radiology Committee Task Force on Quality Assurance Protocol, Report No.4.

- American Association of Physicists Medicine, AAPM, Report No.31 (1991): Standard Methods for Measurements Diagnostic, exposure reduction through quality assurance for diagnostic $\mathrm{x}$-ray procedure, Health phy, volume 5.
- American Association of Physicists in Medicine, AAPM. (2002): Quality Control in Diagnostic Radiology” Report of Task Group \#12, Diagnostic X-ray Imaging Committee.

- Begum, M.; Mollah, A.S.; Zamman, M.A. and Rahman, M. (2011): Quality Control tests in Some Diagnostic X-ray Units in Bangladesh. Bangladesh J. Med Phys., 4(1): 59

- Food and Drug Administration, FDA. (1999): Resource Manual for compliance test parameters of Diagnostic X-ray system.

- Ismail, H.A.; Ali, O.A.; Omer, M.A.; Garelnabi, M.E. and Mustafa, N.S. (2015): Evaluation of Diagnostic Radiology Department in Term of Quality Control (QC) of X-Ray Units at Khartoum State Hospital. Int. J. Sci. Res., 4(1):1875

- Plotti, J.L. (1995): Guidelines for Quality Assurance in Radiation Protection for Diagnostic X-ray Facilities" NRL.

- Stephen, I.; Cyril, S.; Geoffre, E. and John, J.F. (2001): Quality Assurance and Quality Control of Equipment in Diagnostic Radiology Practice, The Ghanaian Experience, Wide Spectra of Quality Control., www.intechopen.com.

- Taha, M.T. (2015): Study the quality Assurance of Coventional X-ray machine using non-invasive KV meter. Int. J. Sci. Res., 4(3):372

- Taha, M.T; Al-Ghorabie, F.H.; Kutbi, R.A and Saib, W.K. (2015): Assessment of Entrance Skin Doses for Patients Undergoing Diagnostic X-Ray Examinations in King Abdullah Medical City. Makkah, KSA. J. Rad. Res. App. Sci., 8(1):100. 